\title{
Phase contrast imaging measurements and numerical simulations of turbulent density fluctuations in gas-fuelled ECRH discharges in Wendelstein 7-X
}

\author{
J.-P. Bähner ${ }^{\circledR 1}{ }^{\dagger}$, J.A. Alcusón ${ }^{\circledR 1}$, S.K. Hansen ${ }^{\circledR 2}$, A. von Stechow ${ }^{\circledR 1}$, \\ O. Grulke ${ }^{1,3}$, T. Windisch ${ }^{1}$, H.M. Smith ${ }^{1}$, Z. Huang ${ }^{2}$, E.M. Edlund ${ }^{4}$,
} M. Porkolab ${ }^{2}$, M.N.A. Beurskens ${ }^{1}$, S.A. Bozhenkov ${ }^{\circledR 1}$, O.P. Ford ${ }^{\circledR} 1$, L. Vanó $^{1}$, A. Langenberg ${ }^{(1)}$, N. Pablant ${ }^{\circledR 5}$, G.G. Plunk ${ }^{\circledR 1}$, A. Bañón Navarro ${ }^{\circledR 6}$, F. Jenko ${ }^{106}$ and The W7-X Team ${ }^{1}$

${ }^{1}$ Max Planck Institute for Plasma Physics, 17491 Greifswald, Germany

${ }^{2}$ MIT Plasma Science and Fusion Center, Cambridge, MA 02139, USA

${ }^{3}$ Technical University of Denmark, 2800 Kengens Lyngby, Denmark

${ }^{4}$ SUNY Cortland, Cortland, NY 13045, USA

${ }^{5}$ Princeton Plasma Physics Laboratory, Princeton, NJ 08540, USA

${ }^{6}$ Max Planck Institute for Plasma Physics, 85748 Garching, Germany

(Received 15 March 2021; revised 26 May 2021; accepted 28 May 2021)

The fundamental nature of turbulent density fluctuations in standard Wendelstein 7-X (W7-X) stellarator discharges is investigated experimentally via phase contrast imaging (PCI) in combination with gyrokinetic simulations with the code GENE. We find that density fluctuations are ion-temperature-gradient-driven and radially localised in the outer half of the plasma. It is shown that the line-integrated PCI measurements cover the right range of wavenumbers and a favourable toroidal and poloidal location to capture some of the strongest density fluctuations in W7-X. Due to the radial localisation of fluctuations, measured wavenumber-frequency spectra exhibit a dominant phase velocity, which can be related to the $E \times B$ rotation velocity at the radial position of a well in the neoclassical radial electric field. The match is robust against variations of heating power and line-integrated density, which is partly due to the localisation of fluctuations and partly due to effects of the radial gradient in the $E \times B$ velocity profile on the wavenumber-frequency spectrum. The latter effect is studied with a newly built synthetic PCI diagnostic and global gyrokinetic simulations with GENE-3D.

Key words: fusion plasma

\section{Introduction}

The Wendelstein 7-X (W7-X) stellarator is optimised for reduced neoclassical transport (Beidler et al. 1990). Results from the first operation phase with an island divertor strongly

$\dagger$ Email address for correspondence: jan-peter.baehner@ipp.mpg.de 
suggest that the optimisation is successful and the neoclassical transport is indeed smaller than in comparable conventional stellarators (Klinger et al. 2019; Smith et al. 2019; Wolf et al. 2019). The majority of discharges were gas fuelled from the edge and externally heated via electron cyclotron resonance heating $(\mathrm{ECRH})$. A significant fraction of the transport in this type of discharge is anomalous (Geiger et al. 2019; Klinger et al. 2019). This finding is supported by the observation of improved energy confinement in scenarios during which turbulence is strongly suppressed, e.g. in the case of central plasma density profile peaking due to pellet injections (Klinger et al. 2019; Wolf et al. 2019). This improvement is associated with a suppression of ion-temperature-gradient (ITG)-driven turbulence (Alcusón et al. 2020; Bozhenkov et al. 2020; Stechow et al. 2021).

Turbulence in magnetised plasmas is usually generated by temperature and density gradient driven electrostatic microinstabilities, the most important ones being the ITG, electron temperature gradient (ETG) and trapped electron mode (TEM) (Doyle et al. 2007). Their respective role for turbulence and transport has been explored analytically and by means of gyrokinetic simulations for stellarators and W7-X in particular (Proll et al. 2012; Proll, Xanthopoulos \& Helander 2013; Proll et al. 2016; Helander, Proll \& Plunk 2013; Plunk et al. 2014; Plunk, Connor \& Helander 2017; Plunk et al. 2019). Besides gyrokinetic simulations with codes like GENE (Jenko et al. 2000), key tools on W7-X to investigate turbulence in the core of the plasma experimentally are diagnostics like phase contrast imaging (PCI) (Edlund et al. 2018; Huang et al. 2021) and Doppler reflectometry (DR) (Carralero et al. 2019; Windisch et al. 2019). Detailed experimental studies of dominant instability evolution in W7-X are limited. This publication focuses on turbulent density fluctuations as measured by the PCI diagnostic. They are compared with expectations of dominant instabilities, and a radial localisation due to the driving gradients is investigated. Gyrokinetic simulations with GENE are employed to study the dominant instabilities, the radial evolution of fluctuations and the poloidal mode structure. The phase velocity of the measured fluctuations is shown to be dominated by the neoclassical $E \times B$ rotation (Doppler shift). Radial localisation of the line-integrated PCI measurement is achieved by matching the measured phase velocity with the $E \times B$ rotation velocity profile from neoclassical simulations. This includes modelling the effect of the $E \times B$ rotation on the measured spectra with a newly built synthetic PCI diagnostic employing a simplified model for plasma rotation on top of global gyrokinetic simulation results by GENE-3D (Maurer et al. 2020).

The paper is organised as follows. The investigated experimental situation is introduced in $\S 2$. In $\S 3$, data measured by PCI is evaluated including a direct comparison of the observed phase velocity in the turbulent spectrum with the neoclassical $E \times B$ velocity and studies with the synthetic PCI diagnostic. Dedicated gyrokinetic simulations were carried out for the analysed experimental situation and are described in $\S 4$. Finally, in $\S 5$, the results and conclusions are discussed and summarised.

\section{Experimental set-up}

We focus on simple gas-fuelled ECRH discharges with hydrogen. There is no direct ion heating and no additional central fuelling by neutral beam or pellet injection. Since most discharges in W7-X have been of this type, it has the best data coverage. Furthermore, it is the envisioned scenario for the near future steady-state experiments at W7-X.

For most of the analysis, we choose a representative reference plasma. Figure 1 shows an overview of the chosen discharge. The average magnetic field strength on the magnetic axis is $\left\langle B_{0}\right\rangle=2.41 \mathrm{~T}$, with a mirror ration of $4.3 \%$ and a rotational transform of $\iota / 2 \pi=0.86$ on the axis and $\iota / 2 \pi=1$ at the edge, forming a 5/5-island structure in the scrape-off-layer (W7-X standard configuration, Geiger et al. 2014). The external heating is purely ECRH 

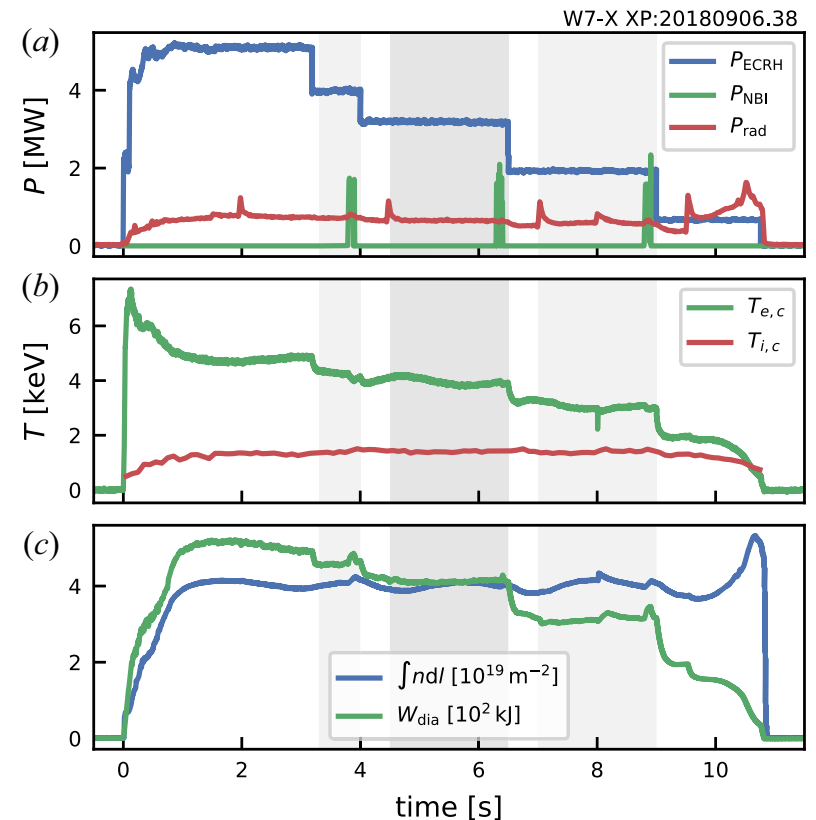

FIGURE 1. Overview of a typical ECRH discharge in W7-X with hydrogen as the main ion species. The input power was stepped down while keeping the density constant. Transient neutral beam injections (NBI) were launched for diagnostic purposes. The depicted electron and ion temperatures correspond to measurements of the central channel of the electron cyclotron emission (known as ECE) diagnostic and the X-ray imaging crystal spectrometer (XICS), respectively.

with short NBI for diagnostic purposes. The heating power is stepped down during the discharge, while the line-integrated density is kept constant. Accordingly, the central electron temperature and diamagnetic energy decrease during the discharge. The ion temperature is constant, which is the case for almost every gas-fuelled ECRH discharge in W7-X (Bozhenkov et al. 2019). During the power steps, which are, respectively, much longer than the energy or particle confinement times, the plasma is stationary. The time window 4.5-6.5 s, with medium heating power, $P_{\mathrm{ECRH}}=3.2 \mathrm{MW}$, (middle shaded region in figure 1) was selected as an experimental reference for the following analyses and simulations. Figure 2 shows the density and temperature profiles of the reference plasma (time average over 4.5-6.5 s), which are paradigmatic for ECRH plasmas in W7-X. The density and ion-temperature profiles are flat throughout the core and decrease only outside $r_{\text {eff }} / a=0.7$, where $a$ is the plasma minor radius and $r_{\text {eff }}$ is the effective plasma radius, which in the context of $\mathrm{W} 7-\mathrm{X}$ is defined via the toroidally averaged poloidal cross-sectional area, $\langle A\rangle$, as $r_{\text {eff }}=\sqrt{\langle A\rangle / \pi}$. The electron temperature is centrally peaked and much higher than the ion temperature in the core because of the central electron heating and weak coupling to ions due to the relatively low plasma density. Figure 2(b) shows the respective normalised gradients. The ITG becomes large in the outer half of the minor radius, while the density gradient stays comparably small, which is a combination that is expected to drive ITG turbulence. The ETG modes should be destabilised further in the core of the plasma.

\section{Measurements}

The PCI diagnostic measures line-integrated electron density fluctuations. An infrared laser scatters off the density fluctuations with a small scattering angle. The scattered 


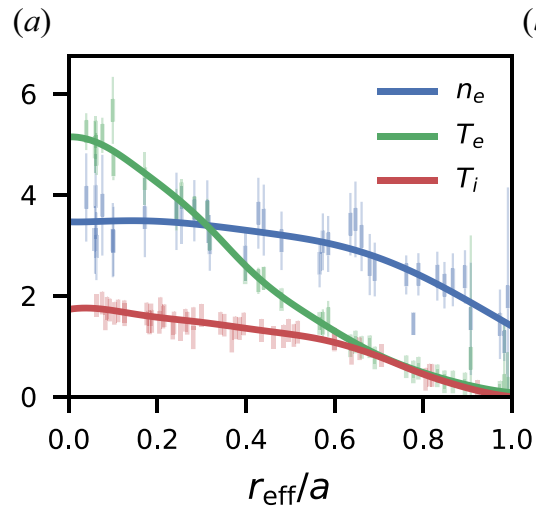

(b)

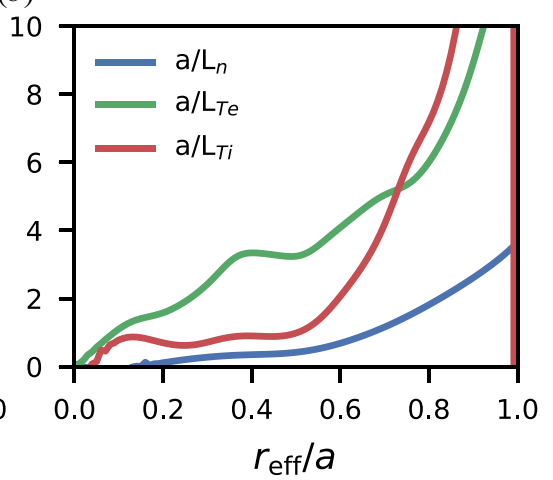

FIGURE 2. Radial profiles of the reference discharge averaged over 4.5-6.5 s. Panel (a) shows the electron density, $n_{e}$ in $10^{19} \mathrm{~m}^{-3}$ and electron temperature, $T_{e}$ in $\mathrm{keV}$, both measured by the Thomson scattering diagnostic, as well as the ion temperature, $T_{i}$ in $\mathrm{keV}$, measured by charge exchange recombination spectroscopy (CXRS) (Ford et al. 2020). The vertical lines are measurement values with error bars which represent measurement uncertainties. The solid lines are best fits to the measurement data. Panel $(b)$ shows the normalised gradient lengths corresponding to the best fits with $L_{X}=-(\mathrm{d} \ln X / \mathrm{d} r)^{-1}$.

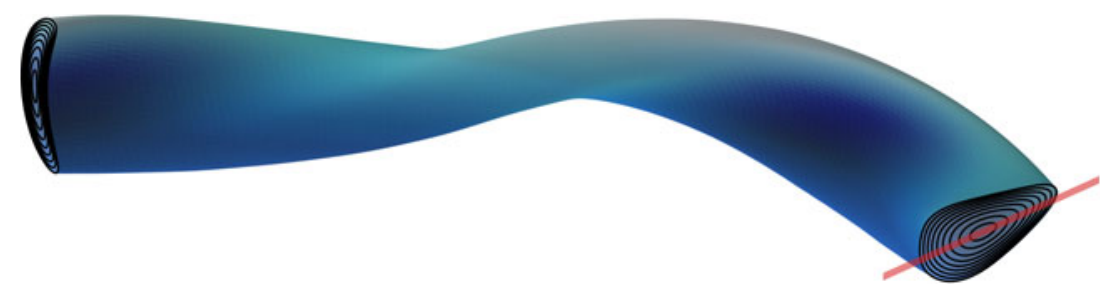

FIGURE 3. Flux surface of $r_{\text {eff }} / a=0.9$ of the W7-X standard magnetic configuration (blue) with the PCI diagnostic line of sights (red) and Poincaré plot of the cross-sections (black) at the toroidal angle of the PCI diagnostic (right, $\phi=261^{\circ}$ ) and the toroidal angle of the so-called bean shape (left, $\phi=\left[0^{\circ}, 72^{\circ}, 144^{\circ}, 216^{\circ}, 288^{\circ}\right]$ ).

light is phase-shifted relative to the main beam and carries only a small fraction of its intensity. By shifting the phase of the main beam and reducing its intensity, the image created at the detector arrays exhibits large enhancement of the components representing the plasma fluctuations (Edlund et al. 2018; Huang et al. 2021). Figure 3 shows a section of W7-X with the so-called bean shape poloidal cross-section (left-hand end) and the cross-section in which the PCI diagnostic measures including the beam path of the infrared laser (right-hand end). The PCI laser beam enters the plasma on the inboard side, intersects the magnetic axis and leaves the plasma on the outboard side. The line-integrated nature of the measurement has the advantage of capturing fluctuations through the entire plasma bulk and thus contains information about turbulence in different regions of the plasma. A radially localised measurement via a selection of certain pitch angles between $B$ and the measured wavenumbers (cf. Dorris, Rost \& Porkolab 2009) is not yet possible but is planned for in the upcoming experimental campaigns (Huang et al. 2021).

The 32 channels of PCI correspond to lines of sight which are configured along a particular spatial direction in the plasma, $\hat{\boldsymbol{e}}_{m}$, such that the measured wavenumber is roughly poloidal. The concurrent temporal and spatial resolution of PCI enables a 


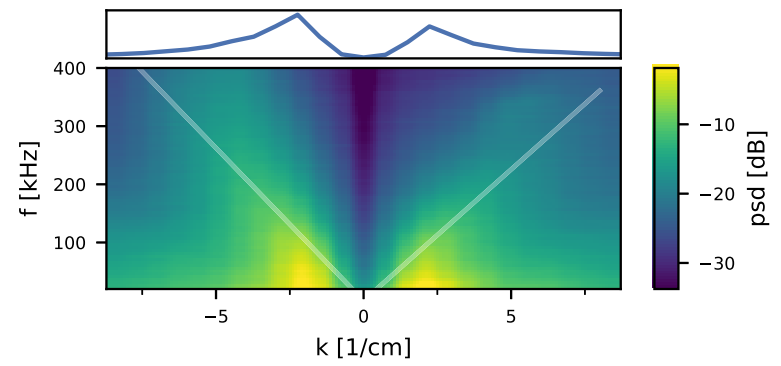

FIGURE 4. Wavenumber-frequency spectrum measured by the PCI diagnostic. The white lines mark the dominant linear phase velocities. In the top panel, the spectral density integrated over the depicted frequency range is shown on a linear scale.

simultaneous frequency and wavenumber analysis of the turbulent fluctuations. First, a complex Fourier transform (FT) in time is performed for each channel, $\hat{s}_{\mathrm{ch}}(f)=$ $\mathrm{FT}\left(s_{\mathrm{ch}}(t)\right)$. A second FT in space along the measurement direction results in a wavenumber-frequency spectrum, $\hat{s}(k, f)$, where $\boldsymbol{k}$ is parallel to $\hat{\boldsymbol{e}}_{m}$. Before every discharge, a sound wave with known amplitude and wavenumber is launched into the beam path outside the plasma vessel and measured with the PCI system for calibration. Furthermore, the frequency response of each individual detector channel is taken into account. This enables measuring absolute density fluctuations as well as accurate wavenumber spectra (Huang et al. 2021). Figure 4 shows the absolutely calibrated wavenumber-frequency spectrum of the reference plasma. At very low wavenumbers, the spectral power is reduced due to an instrumentation cutoff at $k \approx 1.6 \mathrm{~cm}^{-1}$ (Huang et al. 2021). Towards high wavenumbers and high frequencies, the spectral power decreases according to a power law (Stechow et al. 2021). Most of the spectral power is located well below $k=6.6 \mathrm{~cm}^{-1}$, which corresponds to $k \rho_{s}=1$, with the drift scale, $\rho_{s}=c_{\mathrm{si}} / \omega_{\mathrm{ci}}$, evaluated at $r_{\mathrm{eff}} / a=0.75$, where $c_{\mathrm{si}}$ is the ion sound speed and $\omega_{\mathrm{ci}}$ is the ion cyclotron frequency. The wavenumber axis has a positive and a negative branch, which correspond to opposite propagation directions in the laboratory frame. In both branches, a dominant constant phase velocity is observed, i.e. a large fraction of the spectral power is carried by fluctuations which have the same phase velocity in the measurement direction. Negative wavenumber fluctuations have higher spectral power than fluctuations in the positive branch (see the top panel of figure 4).

The phase velocity in the PCI wavenumber-frequency spectrum, $v_{\mathrm{ph}}^{\mathrm{PCI}}$, corresponds to the propagation velocity of the fluctuations in the laboratory frame, $u_{y}$. It is a combination of the poloidal phase velocity of the turbulent modes in the plasma frame, $v_{\mathrm{ph}}$, and the $E \times B$ rotation velocity of the plasma (both in the binormal direction):

$$
u_{y}=v_{\mathrm{ph}}+v_{E \times B} .
$$

It is generally assumed and will be explicitly shown below that the $E \times B$ rotation velocity is much larger than the turbulent phase velocity, such that $u_{y} \approx v_{E \times B}$ (Conway et al. 2011; Windisch et al. 2018; Carralero et al. 2020). The observed dominant linear phase velocity in the PCI wavenumber-frequency spectrum therefore indicates that there is a radial region with little variation of the local $v_{E \times B}$ and strong fluctuations, which cause the spectral power to be concentrated at the corresponding phase velocity. For a given radial profile of $v_{E \times B}$, the radial region can be estimated by directly comparing $v_{\mathrm{ph}}^{\mathrm{PCI}}$ and $v_{E \times B}$. In this interpretation, the two wavenumber branches correspond to the inboard and outboard side, 
since the $E \times B$ rotation changes direction in the frame of the PCI laser beam, when it passes the magnetic axis.

For a quantitative comparison, a consistent way of determining $v_{\mathrm{ph}}^{\mathrm{PCI}}$ from the $k-f$ spectra is necessary. The resulting velocity is sensitive to the selected wavenumber and frequency range, which therefore need to be chosen carefully. A lower boundary of $f \approx 50 \mathrm{kHz}$ was chosen to avoid an interference of the slope with the low- $k$ cutoff. At $k \approx 10 \mathrm{~cm}^{-1}$, a suppression of spectral power was observed (not shown in figure 4), which is attributed to phase scintillation, a persistent optical instrument effect due to minor misalignment of the image plane and the detector. Additionally, wavenumber aliasing can play a role at high wavenumbers and frequencies. Upper boundaries of $k \approx 9 \mathrm{~cm}^{-1}$ and $f \approx 300 \mathrm{kHz}$ are chosen to restrict the analysis to a regime of high confidence, where the exact values are adjusted between discharges. Close to the boundaries, the slope of the dominant phase velocity can appear curved, which is attributed to the described instrument effects rather than a real change of the dominant phase velocity.

Within the chosen wavenumber-frequency regime, the dominant phase velocity is determined as follows. Local maxima of the spectral power along the wavenumber axis, $\max _{\omega=\text { const. }} p s d(k)$, above a certain threshold are determined through cubic spline interpolation. Each maximum corresponds to a phase velocity of a linear dispersion relation, $v_{\mathrm{ph}}=2 \pi f / k$. Since the resolution decreases significantly for high velocities, the maxima are ordered by the angle to the abscissa, $\alpha=\tan ^{-1}\left(v_{\mathrm{ph}}\left(10 \mathrm{~m} \mathrm{~s}^{-1}\right)\right)$, which has a constant resolution (for $\Delta f \approx 1 \mathrm{kHz}$ and $\Delta k \approx 1 \mathrm{~cm}^{-1}$ ). A kernel density estimation $(\mathrm{KDE})$ is calculated to find the angle with the highest density of local maxima in the spectrum. The maximum position of the KDE is finally taken as the dominant phase velocity. This method has the advantage of being able to detect several dominant phase velocities per branch, which appear as secondary peaks in the KDE. Such a situation occurs for example after pellet injection (Stechow et al. 2021) and is not discussed here any further. For the case of the reference plasma (figure 4), this method results in $v_{\mathrm{ph}}^{\mathrm{PCI}}=-3.3 \mathrm{~km} \mathrm{~s}^{-1}$ for the negative wavenumber branch and $v_{\mathrm{ph}}^{\mathrm{PCI}}=2.8 \mathrm{~km} \mathrm{~s}^{-1}$ for the positive wavenumber branch.

\subsection{Neoclassical radial electric field}

In order to obtain a radial profile of $v_{E \times B}$, we compare neoclassical simulations and DR measurements of the background radial electric field, $E_{r}$. In stellarators, it is predominantly determined by the ambipolar neoclassical (known as NC) transport (Helander et al. 2012). The monoenergetic diffusion coefficients for electrons and ions scale differently with the collision frequency, $v$, and have different magnitudes. The ions usually are in the $\sqrt{v}$-regime and the electrons in the $1 / v$-regime (Helander et al. 2012). In order to maintain ambipolarity of the particle fluxes, $\Gamma_{i}=\Gamma_{e}$, a self-consistent radial electric field is established (Mynick \& Hitchon 1983; Yokoyama et al. 2007). For equal ion and electron temperature, the ambipolar electric field is usually negative and compensating for a larger ion particle transport. This situation is called ion root. Due to the strong temperature dependence of the diffusion coefficient in the $1 / \nu$-regime, $D_{e} \propto T_{e}^{7 / 2}$, the electron diffusion increases strongly for local electron heating $\left(T_{e}>T_{i}\right)$ and a second stable solution with a positive $E_{r}$ arises, which is called electron root (Yokoyama et al. 2007; Helander et al. 2012).

Measurements by CXRS and XICS show the existence of an electron root in the core and an ion root in the outer half of ECR heated plasmas in W7-X (Pablant et al. 2018; Ford et al. 2020). They have a generally good agreement with numerical neoclassical calculations by codes like the drift kinetic equation solver, DKES (Hirshman et al. 1986; Turkin et al. 2011). The DR measurements also show a good agreement between the radial electric 


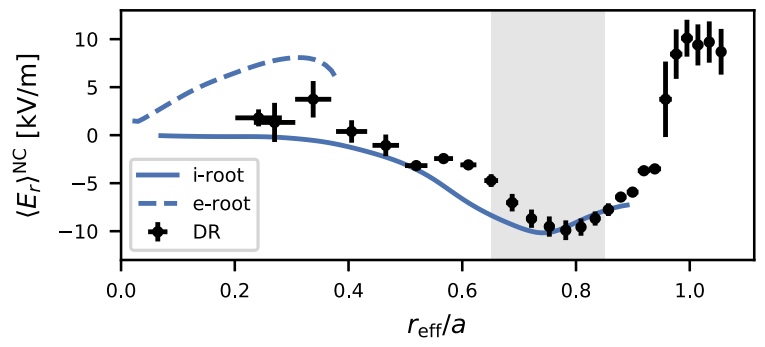

FIGURE 5. Flux-surface averaged neoclassical radial electric field by DKES (blue) and measurements by the Doppler reflectometer (black) for the reference discharge.

field obtained from the poloidal velocity of fluctuations, $E_{r}^{\mathrm{DR}}=u_{y} B$, and neoclassical calculations by DKES (Windisch et al. 2019; Carralero et al. 2020). Figure 5 shows the neoclassical electric field calculated for the profiles of the reference plasma, given in figure 2. At the plasma edge, ion and electron temperatures are set equal where the ion temperature exceeds the electron temperature (since there is no ion heating) and smoothing is applied to avoid a discontinuity in the gradient. The dashed line is the electron-root solution, which only appears in the core, where $T_{e} \gg T_{i}$. The ion root, plotted as a solid line, is relevant in the outer half of the plasma, where it forms an $E_{r}$-well. Sensitivity studies have previously produced an uncertainty of the simulated $E_{r}^{\mathrm{NC}}$ of the order of $10 \%$, by varying the local density and temperature values and gradients by $10 \%$ (Carralero et al. 2020; Estrada et al. 2021). The black error bars are measurement points by DR, which agree well with the ion-root solution by DKES in the region of interest (shadowed region around $75 \%$ of the minor radius, where the drive of ITG turbulence should be large). At the last closed flux surface (known as LCFS), the measured $E_{r}$ changes sign, resulting in a large shear. The corresponding $E \times B$ velocity of the $E_{r}$-well in the region of interest is $v_{E \times B} \approx 4 \mathrm{~km} \mathrm{~s}^{-1}$, which is the same order of magnitude as the phase velocity measured in the PCI wavenumber-frequency spectra. The good agreement of the DR measurement and the simulated $E_{r}$ supports the assumption that the propagation velocity of fluctuations is dominated by the $E \times B$ rotation, $u_{y} \approx v_{E \times B}$.

\subsection{Comparison of velocities}

For a direct comparison of the dominant phase velocity in the PCI wavenumber-frequency spectrum, $v_{\mathrm{ph}}^{\mathrm{PCI}}$, with the local, neoclassical $\boldsymbol{E} \times \boldsymbol{B}$ velocity, geometrical effects along the PCI line of sight have to be taken into account. The PCI measures wavenumbers and velocities along a fixed measurement direction, $\hat{\boldsymbol{e}}_{m}$, which does not strictly correspond to the propagation direction of turbulent fluctuations. The latter is assumed to be perpendicular to the magnetic field direction, $\hat{b}=B / B$, and the flux surface normal, $\hat{\boldsymbol{n}}_{s}=\nabla s /|\nabla s|$

$$
\boldsymbol{u}_{y}=u_{y} \hat{\boldsymbol{n}}_{s} \times \hat{\boldsymbol{b}}
$$

where $s$ is the normalised toroidal flux. The phase velocity measured by PCI is a projection of $\boldsymbol{u}_{y}$ onto the measurement direction,

$$
v_{\mathrm{ph}}^{\mathrm{PCI}}=\boldsymbol{u}_{y} \cdot \hat{\boldsymbol{e}}_{m} .
$$

This projection changes along the line of sight, since both the angle between the magnetic field and the measurement direction as well as the angle between the laser beam and the flux surface normal changes. Measured phase velocities therefore correspond to 
different $u_{y}$ depending on where the fluctuations occur along the line of sight. The local neoclassical electric field is calculated by multiplying the flux surface averaged value of the radial electric field given by DKES, $\left\langle E_{r}\right\rangle^{\mathrm{NC}}$, with $\left|\nabla r_{\mathrm{eff}}\right|$, accounting for the local flux compression. The magnitude of the corresponding $\boldsymbol{E} \times \boldsymbol{B}$ velocity is given by

$$
v_{E \times B}=\frac{\left\langle E_{r}\right\rangle^{\mathrm{NC}}\left|\nabla r_{\mathrm{eff}}\right|}{B} .
$$

Besides the profile of $\left\langle E_{r}\right\rangle^{\mathrm{NC}}$ that was shown in figure 5, the magnetic field magnitude, $B$, as well as $\left|\nabla r_{\text {eff }}\right|$ vary along the PCI line of sight. The geometrical effects described above depend on the exact position of the line of sight and therefore vary slightly across the detector channels. The 32 channels of the detector correspond to 32 parallel lines of sight distributed along $\hat{\boldsymbol{e}}_{m}$ within the width of the laser beam. Since the intensity of the laser across its width has a Gaussian profile, not all channels contribute equally to the wavenumber-frequency spectrum. To illustrate this effect, the local $v_{E \times B}$ and $v_{\mathrm{ph}}$ were calculated for all channel lines of sight and opacity weighted by the respective laser amplitude.

Figure 6 shows the direct comparison of the local $E \times B$ velocity from neoclassical calculations and the local binormal velocity, which corresponds to the measured dominant phase velocity in the PCI wavenumber-frequency spectrum along the PCI line of sight. The $v_{E \times B}$ in the ion diamagnetic direction stems from the electron-root solution for $E_{r}$, the $v_{E \times B}$ in the electron diamagnetic direction from the ion-root solution. Since it has been shown experimentally that an electron-root radial electric field exists in the core, the corresponding electron-root velocity should be preferred where both solutions exist. A typical phase velocity of the ITG modes, $v_{\mathrm{ph}}=500 \mathrm{~m} \mathrm{~s}^{-1}$ in the ion diamagnetic direction, which is later confirmed by linear GENE simulations, is added to $v_{E \times B}$ at $r_{\mathrm{eff}} / a=0.75$ for reference. The negative phase velocity in the PCI wavenumber-frequency spectrum was used for the outboard side mapping and the positive phase velocity for the inboard side (solid lines). The dotted lines correspond to the inverse attribution for the central line of sight. The difference between the 32 lines of sight is very small on the inboard side but relevant on the outboard side. The velocities match best at $r_{\text {eff }} / a \approx 0.75$, which is also the position of the well in $\left\langle E_{r}\right\rangle^{\mathrm{NC}}$. With the inverse attribution, the phase velocity measured by PCI would agree with the neoclassical results only quite close to the core, near the electron-root solution. Since we are looking for the region of strongest fluctuations, we concentrate on the attribution of wavenumber branches to the inboard and outboard side for which the velocities match in the outer half of the plasma, where we expect a larger drive for ion scale turbulence. The turbulent contribution to $u_{y}$ is just a small correction and of the same order as the geometrical effects of the finite beam width and the uncertainty of $v_{E \times B}$ due to the uncertainty of the input profile parameters $(\approx 10 \%$, not shown here). The observed match suggests that strong fluctuations exist at $r_{\text {eff }} / a \approx 0.75$ which cause a dominant linear phase velocity in the PCI wavenumber-frequency spectrum determined by $v_{E \times B}$ at that position.

A simple test for the hypothesis that $v_{\mathrm{ph}}^{\mathrm{PCI}}$ in each wavenumber branch is determined by $v_{E \times B}$, is an experiment with reversed magnetic field direction but otherwise similar plasma parameters. The $\boldsymbol{E} \times \boldsymbol{B}$ rotation changes direction while everything else stays the same, if the discharges are similar enough. We compare two almost identical experiments in a magnetic configuration with lower rotational transform. In both discharges, $5 \mathrm{MW}$ of ECRH were applied at a line-integrated density of $4 \times 10^{19} \mathrm{~m}^{-2}$. Figure 7 shows the wavenumber-frequency spectra and the simulated neoclassical radial electric field of the respective discharges. The radial electric field profiles are very similar. Since $v_{E \times B}$ 


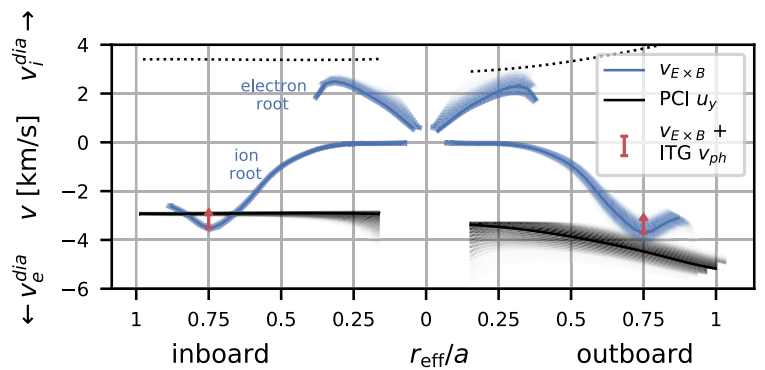

FIGURE 6. Local $\boldsymbol{E} \times \boldsymbol{B}$ rotation velocity from neoclassical calculations and the binormal velocity corresponding to the dominant phase velocity in PCI the spectrum along the line of sight of PCI. A linear estimate of the phase velocity of ITG modes at $r_{\mathrm{eff}} / a=0.75$ (see $\S 4.1$ ) is added to $v_{E \times B}$ for reference. The dotted lines show the alternative attribution of wavenumber branch to inboard/outboard side for the central line of sight. The faded black and blue lines illustrate the geometric variation of the mapping across the 32 lines of sight weighted by the respective laser power, as described in the text.

(a)

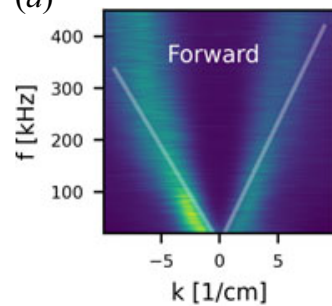

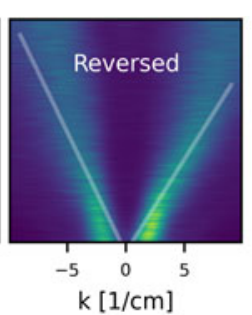

(b)

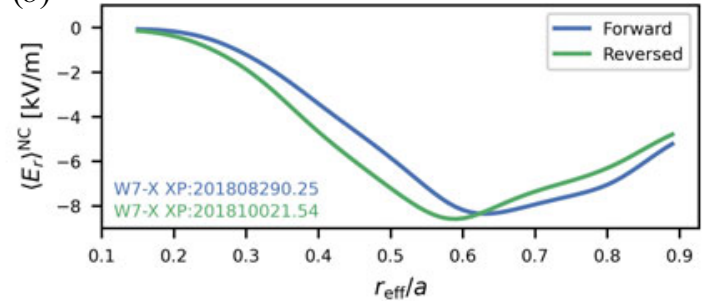

FIGURE 7. (a) Wavenumber-frequency spectrum and dominant phase velocity of discharge with forward and reversed field direction. The spectra are normalised to the mean along the $k$-axis for a better visualisation of the dominant phase velocity. (b) The DKES $E_{r}$ for the respective discharges.

changes direction, the spectrum is mirrored at the $k=0$ axis when going from forward to reversed field, but looks otherwise identical. The measured dominant phase velocities for the negative (positive) wavenumber branch are $v_{\mathrm{ph}}^{\mathrm{PCI}}=-2.3(2.9) \mathrm{km} \mathrm{s}^{-1}$ for the forward field case and $v_{\mathrm{ph}}^{\mathrm{PCI}}=-2.9(2.3) \mathrm{km} \mathrm{s}^{-1}$ for the reversed field case. The asymmetry in the spectral power also switches sides. The velocity comparison yields a similarly good match as in the reference plasma for both cases (see Appendix A), only if the attribution of each $k$-branch to the inboard or outboard side is reversed. There is no electron-root solution in this case, which excludes an inverse attribution. The match is best at the location of the $E_{r}$-well, which is not at $r_{\text {eff }} / a=0.75$ but farther inwards. This poses the question, whether the location of strongest fluctuations is different to the reference case and which role the $E_{r}$-well itself plays for the dominant phase velocity in PCI spectra.

We repeated the analysis for discharges with varying input power or density. Both variations have an effect on the radial location and the depth of the well in the ion root $E_{r}$. For the scan in ECRH power, three time windows within the discharge presented in figure 1 (shaded regions) were analysed, where the ECRH power was stepped down from $4 \mathrm{MW}$ over 3.5 MW to $2 \mathrm{MW}$, while keeping the line-integrated density constant at $4 \times 10^{19} \mathrm{~m}^{-2}$. For the scan of plasma density, three discharges with heating power $P_{\text {ECRH }}=4.7 \mathrm{MW}$ were chosen. The line-integrated density was increased between the 
discharges from $4 \times 10^{19} \mathrm{~m}^{-2}$ over $6 \times 10^{19} \mathrm{~m}^{-2}$ to $8 \times 10^{19} \mathrm{~m}^{-2}$. Details of the velocity comparison for each case can be found in Appendix A. In all situations, the measured dominant phase velocity follows the trend of increasing or decreasing $E_{r}$-well depth. The match is usually best around the position of the $E_{r}$-well, which also varies but only within the outer half of the plasma. In many cases, the electron-root solution is very small or does not exist at all, which excludes the possibility of a significant contribution of core density fluctuations to the dominant phase velocity. We conclude that strong density fluctuations must be present in the outer half of the plasma and that the well in the $v_{E \times B}$-profile, which appears in the same radial region, determines the observed dominant phase velocity in PCI wavenumber-frequency spectra.

While there must be strong fluctuations at the position of the $E_{r}$-well, the form of the $v_{E \times B}$-profile itself must have an impact on the observed wavenumber-frequency spectrum as well. The spectral power originating from fluctuations at a radial location with a strong radial velocity gradient will be spread out in $(k f)$-space. If the fluctuations arise at a radial location with small velocity gradient, the spectral power is focused along a single angle in the wavenumber-frequency spectrum. Fluctuations will produce clearer dominant phase velocities, if they appear in a region in which the velocity profile is flat. While this does not disagree with the interpretation of the strongest density fluctuations causing the dominant phase velocity, both mechanisms have to be taken into account to understand the PCI spectra. In the following subsection, we examine the effect of the velocity gradient on PCI spectra with a synthetic PCI diagnostic (Coda 1997; Lin et al. 2009; Rost, Lin \& Porkolab 2010; Tsujii et al. 2015).

\subsection{Synthetic PCI diagnostic}

A synthetic PCI diagnostic is a useful tool for investigating simplified situations such as different radial gradients of the background velocity field and for comparing simulated three-dimensional density fluctuations with measurement data. It reproduces the phase contrast imaging signal numerically by integrating the $\tilde{n}_{e}$ profile along the PCI line of sight based on the model found in chapter 2 of Coda (1997). This model includes known instrument functions that affect the measurement. The details of the synthetic diagnostic will be presented elsewhere. The primary application in this work is investigating the effect of a radial velocity gradient on the dominant phase velocity in PCI wavenumber-frequency spectra. We implement a simplified model which captures the basic features of the $v_{E \times B}$-profile around the PCI line of sight without the need for a fully self-consistent solution of the problem, since global nonlinear gyrokinetic simulations including the effects of a radial electric field have not been performed at the time of writing. A model density fluctuation background, which is derived from a simulation for W7-X with GENE-3D (Bañón Navarro et al. 2020), is rotated along the poloidal angle according to a predefined rotation velocity. The model is described in more detail in Appendix B. It is important to point out that the investigated effect is different from turbulence suppression by $\boldsymbol{E} \times \boldsymbol{B}$ shear (Burrell 1997). Neither the linear stabilisation of turbulent modes nor the nonlinear decorrelation through $E \times B$ shear can be simulated with the simplified model of the $v_{E \times B}$ rotation. However, a shift of spectral power to higher wavenumbers is possible in the present model through shearing of structures spanning several flux surfaces. In order to test the effect of a velocity gradient, fluctuations were restricted to a narrow radial interval by a top-hat function of width $0.2 a$ centred around $r_{\text {eff }} / a=0.7$. The velocity profiles have the same value at the centre with a constant gradient over the interval as shown in Appendix B. In principle, one would expect the resulting wavenumber-frequency spectra to show the same dominant phase velocity for each case 
(a)

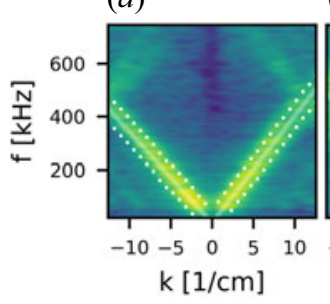

(b)

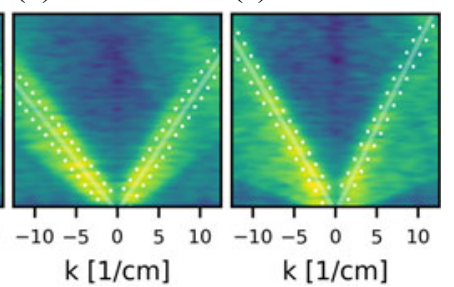

FIGURE 8. Wavenumber-frequency spectra of synthetic PCI measurements corresponding to model velocity profiles with increasing radial gradient from $(a-c)$. The colour scale is in $\mathrm{dB}$. The white lines mark the dominant linear phase velocities, the white dashes mark a $\pm 50 \mathrm{kHz}$ interval around the dominant $v_{\mathrm{ph}}$.

with a varying concentration of spectral power. Figure 8 shows the wavenumber-frequency spectra for three different gradients of the model velocity profile. The radial velocity gradient increases from left to right. In the left-hand case, the gradient corresponds roughly to a rigid plasma rotation and shows a very distinct dominant phase velocity with little spectral power in other parts of the spectrum. Model profiles with even flatter gradient produced very similar results and are therefore not shown here. For increasing gradients, the spectral power is clearly spread out over a larger area in $(k f)$-space. The gradient of the right-hand case corresponds roughly to the maximum gradient that is observed experimentally at the edge of the plasma in discharges without pellet injection, NBI or impurity injection. In order to measure this effect quantitatively, we compare the integrated spectral power in a $\pm 50 \mathrm{kHz}$ band around the dominant phase velocity, $s_{v}$, with the total power in the spectrum, $s_{\text {total }}$. The velocity band is marked by white dashes in figure 8 . In the left-hand case, $68 \%$ of the power is concentrated along the negative $v_{\mathrm{ph}}$ and $31 \%$ along the positive $v_{\text {ph }}$. Only $1 \%$ of spectral power appears outside the velocity bands. For the most severe gradient, the right-hand case, these fractions go down to $50 \%$ and $8 \%$ for the negative and positive velocity band, respectively. Additionally, the dominant phase velocity increases for the cases with higher gradient, even though the average velocity over the top-hat function stays the same. This is because the maximum velocity on the domain of the $\tilde{n}_{e}$ step function increases. Due to the nonlinear relation between phase velocity and the slope in the wavenumber-frequency spectrum, more power is concentrated at a larger slope. In other words, the dominant phase velocity is prone to show the higher end of phase velocities in the fluctuations. These results have important consequences: if the velocity profile has a well, as it does in the experiment, the concentration of spectral power at the slope of the velocity in the well is not only supported by the low gradient at that position but also by the fact that fluctuations with a high velocity appear on the same small area in the $(k f)$-space.

Finally, for a direct comparison with the experimental wavenumber-frequency spectrum, the simulated neoclassical $v_{E \times B}$-profile, taken from figure 5, and a simulated fluctuation amplitude profile, taken from Bañón Navarro et al. (2020), are used as input for the model. Figure 9 shows the resulting wavenumber-frequency spectrum, which has very similar features as the experimental spectrum (figure 4). The dominant phase velocity in the spectrum matches $v_{E \times B}$ at the position of the well on both the inboard and outboard side, which confirms that the analysis is consistent. A more detailed or even quantitative comparison of experimental and simulated spectra is not reasonable, since the simulation in (Bañón Navarro et al. 2020) does not closely match the experimental situation of the reference discharge. Nonlinear global simulations with GENE-3D including the 


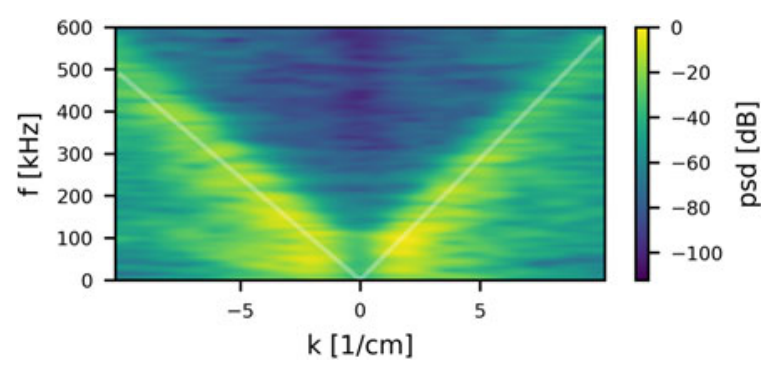

FIGURE 9. Wavenumber-frequency spectrum of synthetic PCI measurement with the simplified model for the $E \times B$ rotation. Density fluctuations from global gyrokinetic simulation (Bañón Navarro et al. 2020) are rotated according to the simulated neoclassical $v_{E \times B}$-profile in figure 5.

background radial electric field are planned, which can then be directly compared with measurement data via the synthetic diagnostic.

\section{Gyrokinetic simulations}

In this section, we present dedicated gyrokinetic simulations for the reference experimental situation, in order to give context to the measurement results and evaluate their compatibility with theoretical and numerical expectations. We use linear simulations to study the dominant instabilities that drive turbulence and nonlinear simulations to investigate the radial and poloidal localisation of density fluctuations as well as the representativity of the PCI measurement for turbulence in general.

\subsection{Linear gyrokinetic flux-tube simulations}

Linear gyrokinetic simulations are used to study the linear drive and dominant mode type in order to identify how turbulence reacts to changes in the density and temperature profiles. We first investigate the dependence of the fundamental modes on the normalised ion-temperature-gradient, $a / L_{T_{i}}$, and the temperature ratio, $\tau=T_{i} / T_{e}$, for W7-X with a scan over those parameters, similar to Zocco et al. (2018). It has been shown that ITG modes in W7-X are most unstable in a narrow toroidal band located at the outboard midplane of the bean shape cross-section, where the curvature is the most unfavourable (Xanthopoulos et al. 2014). A flux-tube at a minor radius of $r_{\text {eff }} / a=0.75$ passing through that region was chosen for the collisionless simulations with kinetic electrons and without electromagnetic effects. The density gradient was chosen according to the value of the experimental profile at this radius $\left(a / L_{n}=1.5\right.$, see figure 2$)$ and a resolution of $\left(n_{x}, n_{y}, n_{z}, n_{v_{\|}}, n_{\mu}\right)=(31,1,128,64,8)$. By including kinetic electrons and a finite density gradient, TEMs are simulated as well and the situation is closer to the experiment. The ETG modes are expected to be unstable for the given experimental electron-temperature-gradient. However, they grow at smaller scales than TEMs and ITG modes and are likely not detected by PCI. They are, consequently, not relevant for the interpretation of the PCI density fluctuation measurements. Additionally, it has been shown that at the chosen radius, where $\tau=1$ in the experiment, the nonlinear contribution of ETG to turbulent transport is negligible compared with ITG and TEM, since the inherently small parallel connection lengths in modular stellarators prevent ETG from forming radially extended streamers (Plunk et al. 2019). We focus on ITG and TEM and neglect ETG modes by assuming $a / L_{T_{e}}=0$. The normalised ion-temperature-gradient length is varied between 1 and 8 for three different temperature ratios. Figure 10 shows 


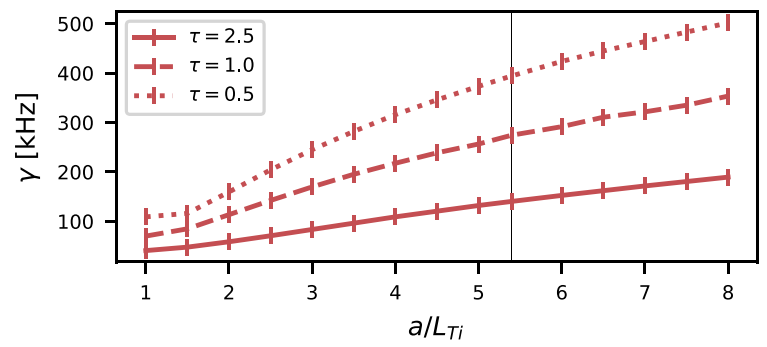

FIGURE 10. Maximum growth rates of simulated wavenumbers for a scan of normalised ion-temperature-gradient lengths and temperature ratios, $\tau=T_{i} / T_{e}$. The vertical line marks the experimental $a / L_{T_{i}}$ at $r_{\mathrm{eff}} / a=0.75$.

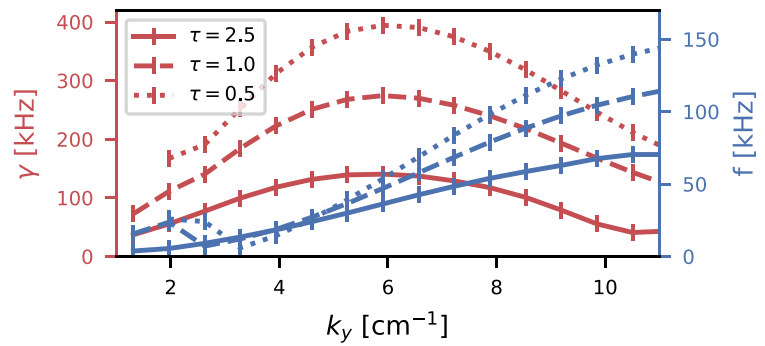

FIGURE 11. Simulated growth rates and real frequencies in the relevant wavenumber range for different temperature ratios at $a / L_{T_{i}}=5.4$.

the linear growth rates of the most unstable modes along the simulated wavenumbers that include ion and intermediate scales $\left(k_{y} \rho_{s} \lesssim 10\right)$ for the described scan. There is a clear positive scaling of the growth rates with $a / L_{T_{i}}$ and a negative scaling with $\tau$, which is typical for ITG modes. In the reference discharge, $a / L_{T_{i}}=5.4$ at this radial location, which is marked by a vertical line in the figure. The experiment situation is far from classical marginal stability, which appears at $a / L_{T_{i}} \approx 1.5$. Figure 11 shows the growth rates and real frequencies versus the binormal wavenumber, $k_{y}$, for three temperature ratios and $a / L_{T_{i}}=5.4$. In order to facilitate a direct comparison with the experimental data of PCI, the quantities in figures 10 and 11 are shown in real units, which are obtained from the normalised simulation output with the temperature and density at $r_{\text {eff }} / a=0.75$ according to the experimental profiles (figure 2) and the local magnetic field at that position. The most unstable wavenumber is around $k_{y}=6 \mathrm{~cm}^{-1}$ for all considered temperature ratios, which is slightly below $k_{y} \rho_{s}=1$, with the ion drift scale, $\rho_{s}$, as expected for ITG and TEM modes. At this wavenumber range, the real frequencies show a linear scaling with $k_{y}$. Together with the clear scaling with $a / L_{T_{i}}$, which can also be observed for the real frequencies over most of the wavenumber range, this suggests that the underlying modes are resonant toroidal ITG modes. The TEMs appear to play a minor role, since the presented characteristics are clearly ITG-like. Pure TEMs propagate in the electron diamagnetic direction, which is expressed by negative frequencies in GENE and not seen in figure 11. Additional flux-tube simulations of pure ITG modes $\left(a / L_{n}=0\right)$ and pure TEMs $\left(a / L_{T_{i}}=0\right)$ confirms that the TEM growth rates are much smaller than those of pure ITG or the combined case for ion- and intermediate scales. On the one hand, this can be attributed to the comparably small density gradient, on the other hand, it has been shown that TEMs are inherently suppressed in quasi-isodynamic magnetic configurations 


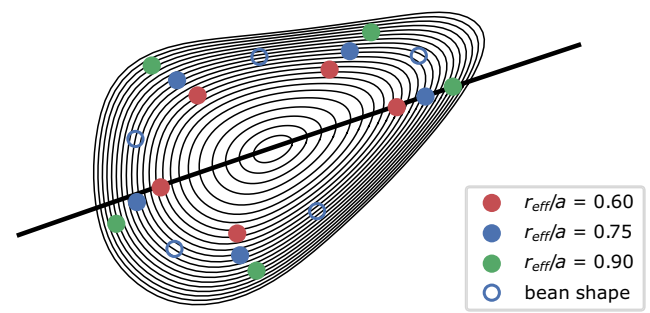

FIGURE 12. Intersections of the GENE flux-tubes with the cross-section of the PCI line of sight (thick black line).

such as W7-X (Proll et al. 2012, 2016). A moderate increase of $a / L_{n}$ is likely to have an overall stabilising effect as it suppresses ITG before TEMs become relevant (Alcusón et al. 2020). We conclude that the turbulence at the chosen location in the reference discharge is determined by toroidal ITG modes, with the observed implication for the effect of $\tau$ and $a / L_{T_{i}}$ on the modes. Even though there is usually a shift in the wavenumber range between linear and nonlinear simulation results, PCI generally seems to cover the right wavenumber range to observe the dominant modes. With the given frequencies at the most unstable wavenumbers, those modes have a phase velocity of $v_{\mathrm{ph}} \approx 500 \mathrm{~m} \mathrm{~s}^{-1}$ in the ion diamagnetic direction, which has been used in figure 6 as a reference and confirms that the phase velocity of turbulent modes is much smaller than the neoclassical $v_{E \times B}$.

\subsection{Nonlinear gyrokinetic flux-tube simulations}

Nonlinear gyrokinetic simulations are the best tool available to investigate the turbulent fluctuations that contribute to transport. Fully three-dimensional nonlinear gyrokinetic simulations of the plasma are still at an early stage of their development and prohibitively expensive. In order to study the radial and poloidal distribution of density fluctuations, we select four different flux-tubes with a resolution of $\left(n_{x}, n_{y}, n_{z}, n_{v_{\|}}, n_{\mu}\right)=$ $(128,64,128,32,9)$ and a box of $\left(L_{x}, L_{y}, L_{z}\right)=(125,125,2 \pi)$ in normalised GENE units at three radial positions outside the half-radius for nonlinear flux-tube simulations with GENE. Three flux-tubes were chosen such that they intersect the PCI line of sight on the outboard side and simulate the turbulence measured by PCI as close as possible. The fluctuations on the inboard side are also captured by the same flux-tubes, since they pass through the stellarator symmetric PCI cross-section in each of the five identical modules of W7-X and very close to the inboard line of sight position in one of them. Figure 12 shows the flux-tubes at the toroidal angle of PCI. The intersection with the line of sight can be observed on the outboard (right-hand) and inboard (left-hand) side. At $r_{\text {eff }} / a=0.75$, an additional flux-tube was selected, which passes through the outboard midplane in the bean shape cross-section of W7-X (and is hence labelled 'bean shape'). It provides a comparison to assess how representative the PCI density fluctuation measurements are for the strongest fluctuations of this flux surface. It is poloidally shifted to the PCI flux-tube and does not intersect the PCI line of sight. The density and temperature gradients, as well as the temperature ratio for each radial position are listed in table 1 and represent the profiles shown in figure 2 . In the edge plasma, $r_{\mathrm{eff}} / a>0.7$, electron and ion temperatures are equal due to good collisional coupling of the two species. The temperature ratio in this region is therefore assumed to be $\tau \approx 1$. Both species were treated kinetically and without collisions, no electromagnetic effects were included. Figure 13 shows the simulation results for the local density fluctuations along the PCI flux-tube as well as the bean shape flux-tube at the same radial location, $r_{\text {eff }} / a=0.75$, where the brackets, $\langle\cdots\rangle$, denote an average in time and perpendicular directions. The fluctuation amplitudes are very similar, 


$\begin{array}{ccccc}r_{\mathrm{eff}} / a & a / L_{n} & a / L_{T_{i}} & a / L_{T_{e}} & T_{e} / T_{i} \\ 0.60 & 0.7 & 2.1 & 4.1 & 1.2 \\ 0.75 & 1.5 & 5.4 & 5.4 & 1.0 \\ 0.90 & 2.6 & 8.9 & 8.9 & 1.0\end{array}$

TABLE 1. Input parameters for GENE flux-tube simulations. The values are taken from experimental profiles in figure 2. Outside $r_{\mathrm{eff}} / a=0.7$ the ion temperature and electron temperature are assumed to be equal.

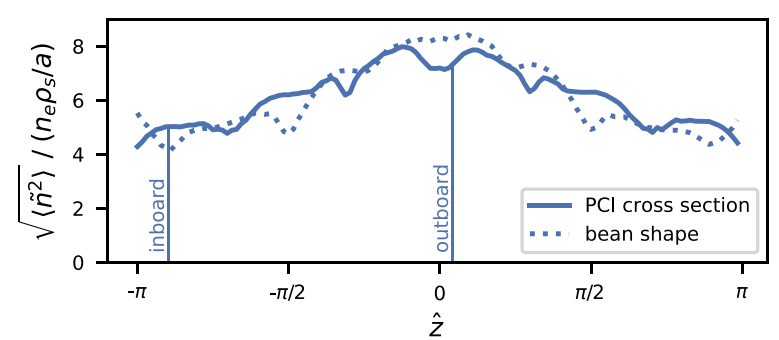

FIGURE 13. Local density fluctuations along the two selected flux-tubes at $r_{\mathrm{eff}} / a=0.75$ from nonlinear GENE simulations. The vertical lines mark the intersections with the PCI line of sight for the solid line. Here $\hat{z}=0$ is defined at the outboard midplane and $\hat{z}= \pm \pi$ at the inboard midplane.

despite the poloidal shift between the two flux-tubes. The poloidal shift means that the same values of $\hat{z}$ correspond to different toroidal positions and the fluctuations might not correlate between the two flux-tubes. The outboard side of the PCI line of sight probes the flux-tube close to the maximum of the mode structure, and the inboard side close to its minimum. The simulated density fluctuations in the flux-tubes at $r_{\text {eff }} / a=0.6$ and $r_{\text {eff }} / a=0.9$ have a rather flat mode structure in the parallel direction and the difference between the PCI inboard and outboard position is negligible. A possible reason for this is the fact that the ITG is either not as strongly driven or suppressed by the increasing density gradient and the band of high fluctuation amplitude (Xanthopoulos et al. 2014), which is a feature of ITG turbulence in W7-X, is not as pronounced as in the strongly driven case at $r_{\mathrm{eff}} / a=0.75$. The band of high fluctuation amplitude at the outboard midplane of the bean shape is wide enough to cover the PCI flux-tube as well and the PCI measurements are a good representation of the general fluctuation level in the stellarator. The asymmetry between the inboard and outboard side fits the experimental observation in the wavenumber-frequency spectrum.

Figure 14 shows the density fluctuations as well as the ion-temperature-gradient for each flux-tube versus the radial position. The density fluctuations are evaluated at the intersection with the PCI line of sight on the inboard and outboard side, but the respective values only show a clear difference at $r_{\text {eff }} / a=0.75$. Both density fluctuations and ion-temperature-gradient are strongest in the $r_{\text {eff }} / a=0.75$ flux-tube and decrease again towards the separatrix, which shows how closely the nonlinear density fluctuations relate to the linear drive of the ITG. This is the numerical confirmation that the fluctuations are indeed strongest at $r_{\text {eff }} / a=0.75$, as it was already derived from the comparison of the dominant phase velocity in the PCI measurement to the neoclassical $v_{E \times B}$. 


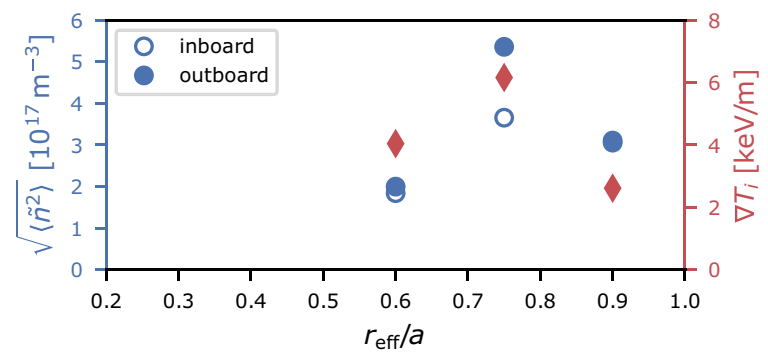

FIGURE 14. Local density fluctuations from nonlinear GENE flux-tube simulations and ion-temperature-gradient at the corresponding radial position. The density fluctuations are evaluated at the intersection with the PCI line of sight on the inboard and outboard side, respectively.

\section{Discussion and conclusion}

We find that radially localised ITG-driven turbulence at the $E_{r}$-well around $r_{\text {eff }} / a=0.75$ characterise $\mathrm{W} 7-\mathrm{X}$ density fluctuations in regular discharge scenarios. The dominance of ITG modes has been shown by linear gyrokinetic simulations and is supported by the experimental observation that the fluctuations are predominantly measured in a wavenumber regime which is typical for ITG turbulence. The radial localisation to $r_{\mathrm{eff}} / a \approx 0.75$ is a result of nonlinear gyrokinetic simulations as well as an extensive comparison of the dominant phase velocity in measured wavenumber-frequency spectra with the neoclassical $E \times B$ rotation velocity, which dominates over the phase velocity of the turbulent modes. This has been shown in linear gyrokinetic simulations and is supported by independent velocity measurements, which agree well with the ambipolar neoclassical electric field. An asymmetry of fluctuation amplitude between the inboard and outboard side has been observed experimentally in the PCI wavenumber-frequency spectrum and in the nonlinear simulations, and agrees with previous flux surface and global simulations (Xanthopoulos et al. 2014; Bañón Navarro et al. 2020). The PCI measurements, although toroidally and poloidally localised and line-integrated, are a good representation of fluctuations in W7-X. Phase contrast imaging covers a suitable range of wavenumbers to measure the dominant ITG turbulence, and captures some of the strongest fluctuations in the whole plasma. The comparison of velocities shows a robust match at the location of the $E_{r}$-well for different discharge scenarios. A new synthetic PCI diagnostic utilises a simplified plasma rotation model and fluctuations from global GENE-3D simulations to show that this is in part due to the effect of radial gradients of the velocity profile on the dominant phase velocity. The effect of the radial electric field on the turbulence through shear stabilisation and decorrelation (Burrell 1997) is not considered, but expected to be small. The $\boldsymbol{E} \times \boldsymbol{B}$ shearing rate after Kinsey, Waltz \& Candy (2005) is almost an order of magnitude smaller than the simulated growth rates in this type of discharge, which suggests that $\boldsymbol{E} \times \boldsymbol{B}$ shear stabilisation is not effective. However, it has recently been shown that the radial electric field itself can reduce turbulence by shifting the band of strong fluctuations out of the region of strong unfavourable curvature (Xanthopoulos et al. 2020).

Since the phase velocity of fluctuations is strongly dominated by the Doppler shift of the $E \times B$ rotation, it does not provide much insight for the underlying turbulence by itself. Other observables such as the wavenumber spectrum or the fluctuation amplitude, however, are largely unaffected by the $E \times B$ rotation. Additionally, there are situations such as the post pellet enhanced confinement phase (Stechow et al. 2021), where the 
wavenumber-frequency spectrum changes dramatically. It will be the subject of future work to determine whether this is rather due to the change of the $\boldsymbol{E} \times \boldsymbol{B}$ velocity profile or a change of the radial location of fluctuations. In this case, the measured phase velocity is a good indicator for a significant change of the turbulence background compared with the standard situation, which is analysed in this work.

Overall, this work has significantly improved the understanding of PCI measurements in W7-X. Many assumptions could be confirmed by experimental data and simulations, which builds a good basis for upcoming analyses and experiments. In particular, the relative importance of neoclassical and turbulent transport is of special interest in W7-X and a localisation of fluctuations in a region where the neoclassic transport is particularly small (Bozhenkov et al. 2020) is an important part of understanding the role of turbulence for transport in W7-X. In the experiments so far, it has been shown that in order to control the ion heat transport, which is key for reaching fusion relevant scenarios, the turbulent transport has to be understood and controlled (Bozhenkov et al. 2020). Developing operation scenarios with improved confinement for W7-X, and eventually a future stellarator reactor, relies on the confidence in gyrokinetic simulations and measurements, which this work aims to improve.

Future work includes investigation of $E_{r}$-effects with global simulations by GENE-3D including a background radial electric field and the synthetic PCI diagnostic, as well as studies with mask filters in PCI for a selective measurement of different radial regions in the upcoming experimental campaigns (Huang et al. 2021).

\section{Acknowledgements}

The GENE simulations have been conducted on the Marconi supercomputer (Italy) and on the Draco supercomputer (Garching, Germany).

Editor Alex Schekochihin thanks the referees for their advice in evaluating this article.

\section{Funding}

This work is partly sponsored by the US Department of Energy, Office of Fusion Energy Sciences under grant number DE-SC0014229. This work has been carried out within the framework of the EUROfusion Consortium and has received funding from the Euratom research and training programme 2014-2018 and 2019-2020 under grant agreement no. 633053. The views and opinions expressed herein do not necessarily reflect those of the European Commission. S.K. Hansen acknowledges support by an Internationalisation Fellowship (CF19-0738) from the Carlsberg Foundation.

\section{Declaration of interests}

The authors report no conflict of interest.

\section{Appendix A. Velocity comparison}

A comparison of the local $\boldsymbol{E} \times \boldsymbol{B}$ rotation velocity from neoclassical calculations with the dominant phase velocity in PCI wavenumber-frequency spectra mapped to the central line of sight of PCI, was performed for various discharges. The discharges differed in ECRH input power, line-integrated density or magnetic field direction. Only the ion-root solution of the neoclassical radial electric field is shown.

Figure 15 shows the velocity comparison for two discharges with forward and reversed magnetic field direction, which is described in $\S 3.2$. Density and temperature profiles for the DKES simulation were measured by the Thomson scattering diagnostic and XICS. 


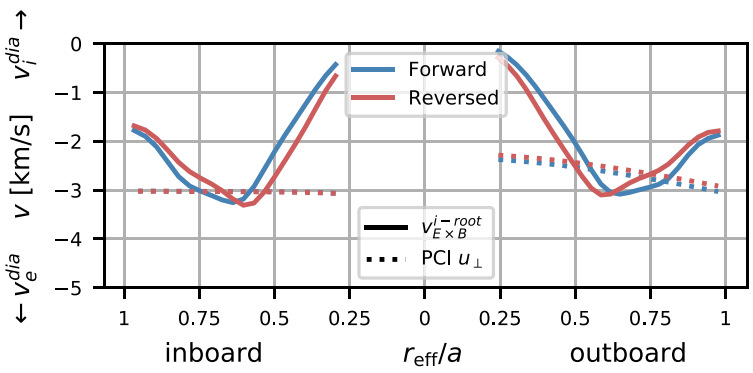

FIGURE 15. Velocity comparison for regular and reversed magnetic field direction.

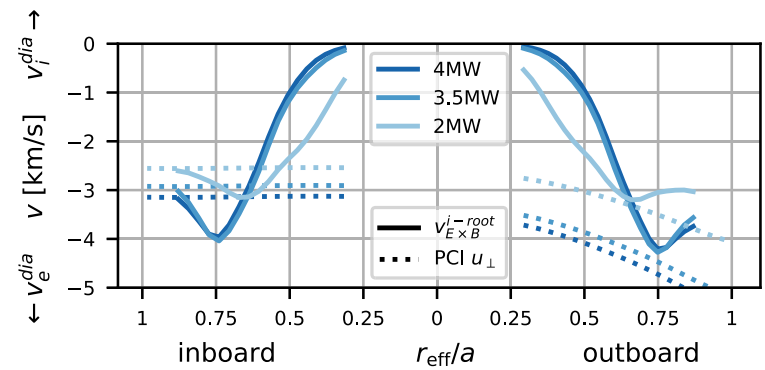

FIGURE 16. Velocity comparison for a scan of ECRH power. The different colours represent values of $P_{\mathrm{ECRH}}$. Electron-root solutions for $v_{E \times B}$ are not shown or do not exist.

For the scan in ECRH power, three time windows within the discharge presented in figure 1 (shaded regions) were analysed, where the ECRH power was stepped down from $4 \mathrm{MW}$ over 3.5 MW to $2 \mathrm{MW}$, while keeping the line-integrated density constant at $4 \times 10^{19} \mathrm{~m}^{-2}$. Density and temperature profiles were measured by the Thomson scattering diagnostic and CXRS. The change in electron heating corresponds directly to a change in central $T_{e}$. The $T_{i}$-profile stays unchanged throughout the discharge. The difference between $T_{e}$ and $T_{i}$ decreases and the radial location at which the two species are in thermal equilibrium moves inwards. This leads to a less significant electron root $E_{r}$, which is confined to a smaller region in the core. The $E_{r}$-well of the ion root becomes less deep and moves radially inwards as well. The comparison with $v_{\mathrm{ph}}^{\mathrm{PCI}}$ is shown in figure 16.

For the scan of plasma density, three discharges with heating power $P_{\mathrm{ECRH}}=4.7 \mathrm{MW}$ were chosen. The line-integrated density was increased between the discharges from $4 \times 10^{19} \mathrm{~m}^{-2}$ over $6 \times 10^{19} \mathrm{~m}^{-2}$ to $8 \times 10^{19} \mathrm{~m}^{-2}$. Density and temperature profiles were measured by the Thomson scattering diagnostic and CXRS. Since the heating power is unchanged, less power is available per particle and $T_{e}$ decreases for the increasing plasma density. The $T_{i}$-profile is identical between the discharges. Similarly to the power step down, the electron root is confined to a smaller region in the core for increasing density. The $E_{r}$-well of the ion root in the outer half of the minor radius decreases in depth for increasing plasma density. The comparison with $v_{\mathrm{ph}}^{\mathrm{PCI}}$ is shown in figure 17.

\section{Appendix B. Simplified $E \times B$ rotation model for the synthetic PCI diagnostic}

The model takes a background $\tilde{n}_{e}$ profile defined on a regular PEST $\left(\psi, \theta^{*}, \varphi\right)$ grid (Maurer et al. 2020), referred to as $\tilde{n}_{e 0}$, from a single toroidal angle, $\varphi$, and evolves the poloidal PEST angle, $\theta^{*}$, to mimic the effect of the radially resolved $\boldsymbol{E} \times \boldsymbol{B}$ rotation. The evolution at different values of the radial PEST coordinate, $\psi$, is considered independently. 


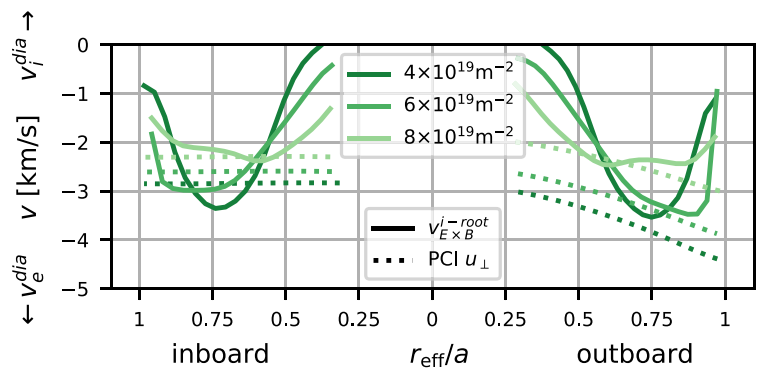

FIGURE 17. Velocity comparison for a scan of line-integrated density. The different colours represent values of $\int n \mathrm{~d} l$. Electron-root solutions for $v_{E \times B}$, if they exist, are not shown.

Referring to the evolved $\theta^{*}$ as $\theta_{\text {arg }}^{*}$, we model the electron density fluctuations by $\tilde{n}_{e}\left(\psi, \theta^{*}, t\right)=\tilde{n}_{e 0}\left(\psi, \theta_{\text {arg }}^{*}(t)\right)$ with $\theta_{\text {arg }}^{*}(0)=\theta^{*}$, where

$$
\frac{\mathrm{d} \theta_{\mathrm{arg}}^{*}}{\mathrm{~d} t}=-\omega\left(\psi, \theta_{\mathrm{arg}}^{*}\right)
$$

and $\omega$ is an effective angular velocity profile given as

$$
\omega=\frac{v_{\theta^{*}}}{\partial \ell / \partial \theta^{*}},
$$

where $v_{\theta^{*}}$ is the projection of $v_{E \times B}$ along the $\theta^{*}$ direction and $\partial \ell / \partial \theta^{*}$ is the derivative of the arclength along the flux surface with respect to $\theta^{*}$. Since we are only interested in the $v_{E \times B}$ profile along the PCI line of sight, we extract values of $\omega$ along the line of sight according to (B2) and map these to the $\psi$ grid on the inboard and outboard sides of W7-X. From this we obtain $\omega\left(\psi, \theta_{\text {in }}^{*}(\psi)\right)=\omega_{\text {in }}(\psi)$ and $\omega\left(\psi, \theta_{\text {out }}^{*}(\psi)\right)=\omega_{\text {out }}(\psi)$, with $\omega_{\text {in }}, \omega_{\text {out }}$ being the $\omega$ values along the PCI line of sight on the inboard and outboard sides, respectively, while $\theta_{\mathrm{in}}^{*}, \theta_{\text {out }}^{*}$ are the corresponding $\theta^{*}$ values. To calculate $\theta_{\mathrm{arg}}^{*}$, we fit the $\omega$ profile by

$$
\omega\left(\psi, \theta^{*}\right)=C(\psi)+B(\psi) \cos \left[\theta^{*}-\theta_{\text {out }}^{*}(\psi)\right]
$$

where

$$
\begin{gathered}
C=\frac{\omega_{\text {in }}-\omega_{\text {out }} \cos \left(\theta_{\text {in }}^{*}-\theta_{\text {out }}^{*}\right)}{1-\cos \left(\theta_{\text {in }}^{*}-\theta_{\text {out }}^{*}\right)}, \\
B=\frac{\omega_{\text {out }}-\omega_{\text {in }}}{1-\cos \left(\theta_{\text {in }}^{*}-\theta_{\text {out }}^{*}\right)},
\end{gathered}
$$

which matches the $\omega$ values along the PCI line of sight. Plugging (B3) into (B1) with the initial condition $\theta_{\mathrm{arg}}^{*}(0)=\theta^{*}$, assuming that $|C|>|B|$, i.e. that the direction of rotation 


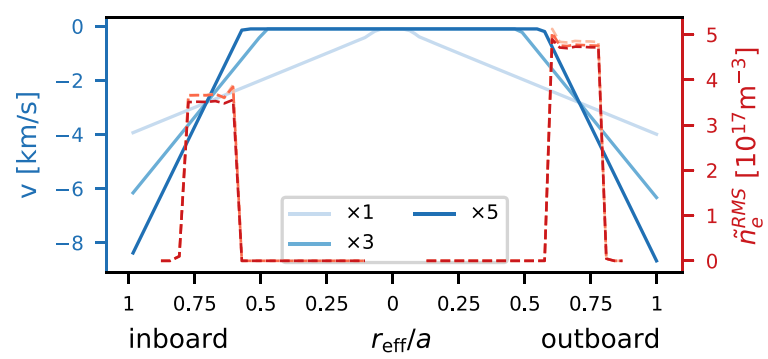

FIGURE 18. Model functions for the $v_{E \times B}$-profile (blue) and corresponding density fluctuation amplitude profile (red) used as input for the synthetic PCI.

does not change sign on a flux surface, now yields

$$
\begin{gathered}
\theta_{\text {arg }}^{*}=\theta_{\text {out }}^{*}+2 \arctan \left(\sqrt { \frac { C + B } { C - B } } \operatorname { t a n } \left[-\operatorname{sign}(C) \frac{\sqrt{C^{2}-B^{2}}}{2} t\right.\right. \\
\left.\left.+\arctan \left\{\sqrt{\frac{C-B}{C+B}} \tan \left(\frac{\theta^{*}-\theta_{\text {out }}^{*}}{2}\right)\right\}\right]\right),
\end{gathered}
$$

allowing calculation of $\tilde{n}_{e}=\tilde{n}_{e 0}\left(\psi, \theta_{\text {arg }}^{*}\right)$ according to the model for arbitrary $\tilde{n}_{e 0}$ and $v_{E \times B}$ profiles. The base $\tilde{n}_{e 0}$ used here is a single time point taken from a simulation performed with the fully three-dimensional, global, nonlinear gyrokinetic code GENE-3D, specifically the adiabatic electron simulation of the W7-X standard magnetic configuration described in Bañón Navarro et al. (2020).

Figure 18 shows the model functions for $v_{E \times B}$ and the density fluctuation amplitude profiles that were used to investigate the effect of steep radial gradients as described in $\S 3.3$. The density fluctuations are restricted to a narrow radial region with a top-hat function. The underlying $\tilde{n}_{e 0}$ had to be scaled individually for each velocity profile for the profile of root mean square values of the rotating fluctuations to follow the same top-hat function. The $v_{E \times B}$-profiles are designed to have the same value at the centre of the fluctuations, while having a different gradient. The profile labelled $\times 1$ corresponds roughly to a rigid plasma rotation, $v_{E \times B} \propto r_{\text {eff }}$. The $\times 5$ profile has five times the radial gradient of the rigid plasma rotation profile, which corresponds roughly to the maximum gradient that is observed experimentally at the edge of the plasma in discharges without pellet injection, NBI or impurity injection.

\section{REFERENCES}

Alcusón, J.A., Xanthopoulos, P., Plunk, G.G., Helander, P., Wilms, F., Turkin, Y., von STECHOW, A. \& GRULKe, O. 2020 Suppression of electrostatic micro-instabilities in maximum- $J$ stellarators. Plasma Phys. Control. Fusion 62 (3), 035005.

Bañón Navarro, A., Merlo, G., Plunk, G.G., Xanthopoulos, P., von Stechow, A., Di Siena, A., Maurer, M., Hindenlang, F., Wilms, F. \& Jenko, F. 2020 Global gyrokinetic simulations of ITG turbulence in the magnetic configuration space of the Wendelstein 7-X stellarator. Plasma Phys. Control. Fusion 62, 105005.

Beidler, C., Grieger, G., Herrnegger, F., Harmeyer, E., Kisslinger, J., Lotz, W., Matssberg, H., Merkel, P., NÜhrenberg, J., Rau, F., et al. 1990 Physics and Engineering Design for Wendelstein VII-X. Fusion Technol. 17 (1), 148-168.

Bozhenkov, S.A., Kazakov, Y., Ford, O., Beurskens, M., Baldzuhn, J., Damm, H., Fuchert, G., Langenberg, A., Pablant, N. \& Pasch, E. 2019 Plasma performance in 
high-density and high-confinement regimes in Wendelstein 7-X. In 46th EPS Conference on Plasma Physics (ed. C. Riconda et al.). European Physical Society (EPS).

Bozhenkov, S.A., Kazakov, Y., Ford, O.P., Beurskens, M.N.A., Alcusón, J., Alonso, J.A., BALDZUhn, J., BRANDT, C., BRUNnER, K.J., DAMm, H., et al. 2020 High-performance plasmas after pellet injections in Wendelstein 7-X. Nucl. Fusion 60 (6), 066011.

BURRELL, K.H. 1997 Effects of ExB velocity shear and magnetic shear on turbulence and transport in magnetic confinement devices. Phys. Plasmas 4 (5), 1499-1518.

Carralero, D., Estrada, T., Windisch, T., Velasco, J.l., Alonso, J.A., Beurskens, M., Bozhenkov, S., DAmm, H., Fuchert, G., GaO, Y., et al. 2020 Characterization of the radial electric field and edge velocity shear in Wendelstein 7-X. Nucl. Fusion 60 (10), 106019.

Carralero, D., Estrada, T., Windisch, T., Velasco, J.L., Alonso, J.A., Beurskens, M., Bozhenkov, S., Damm, H., Fuchert, G. \& Pablant, N. 2019 First V-band doppler reflectometer results from the OP1.2b campaign in Wendelstein 7-X. In Proc. 14th Intl. Reflectometry Wksh. - IRW14 (Lausanne, May 2019) (ed. G.D. Conway). Swiss Plasma Center EPFL.

CODA, S. 1997 An experimental study of turbulence by phase-contrast imaging in the DIII-D tokamak. $\mathrm{PhD}$ thesis, Massachusetts Institute of Technology, Cambridge.

Conway, G.D., Angioni, C., Ryter, F., SAuter, P. \& Vicente, J. 2011 Mea $n$ and oscillating plasma flows and turbulence interactions across the $L-H$ confinement transition. Phys. Rev. Lett. 106, 065001 .

Dorris, J.R., Rost, J.C. \& Porkolab, M. 2009 Localized measurement of short wavelength plasma fluctuations with the DIII-D phase contrast imaging diagnostic. Rev. Sci. Instrum. 80 (2), 023503.

Doyle, E.J., Houlberg, W.A., Kamada, Y., Mukhovatov, V., Osborne, T.H., Polevoi, A., Bateman, G., Connor, J. W, Cordey, J. G., Fujita, T., et al. 2007 Chapter 2: Plasma confinement and transport. Nucl. Fusion 47 (6), S18-S127.

Edlund, E.M., Porkolab, M., Huang, Z., Grulke, O., Böttger, L.-G., von Sehren, C. \& VON STECHOW, A. 2018 Overview of the Wendelstein 7-X phase contrast imaging diagnostic. Rev. Sci. Instrum. 89 (10), 10E105.

Estrada, T., Carralero, D., Windisch, T., Sánchez, E., García-Regaña, J.M., Martínez-Fernández, J., de la Peña, A., Velasco, J.L., Alonso, J.A., Beurskens, M., et al. 2021 Radial electric field and density fluctuations measured by Doppler reflectometry during the post-pellet enhanced confinement phase in W7-X. Nucl. Fusion 61 (4), 046008.

Ford, O.P., Vanó, L., Alonso, J.A., Baldzuhn, J., Beurskens, M.N.A., Biedermann, C., Bozhenkov, S.A., Fuchert, G., Geiger, B., Hartmann, D., et al. 2020 Charge exchange recombination spectroscopy at Wendelstein 7-X. Rev. Sci. Instrum. 91 (2), 023507.

Geiger, B., Wegner, T., Beidler, C.D., Burhenn, R., Buttenschön, B., Dux, R., Langenberg, A., Pablant, N.A., PÜtterich, T., Turkin, Y., et al. 2019 Observation of anomalous impurity transport during low-density experiments in W7-X with laser blow-off injections of iron. Nucl. Fusion 59 (4), 046009.

Geiger, J., Beidler, C.D., Feng, Y., MaAßBerg, H., Marushchenko, N.B. \& Turkin, Y. 2014 Physics in the magnetic configuration space of W7-X. Plasma Phys. Control. Fusion 57 (1), 014004.

Helander, P., Beidler, C.D., Bird, T.M., Drevlak, M., Feng, Y., Hatzky, R., Jenko, F., Kleiber, R., Proll, J.H.E., Turkin, Y., et al. 2012 Stellarator and tokamak plasmas: a comparison. Plasma Phys. Control. Fusion 54 (12), 124009.

Helander, P., Proll, J.H.E. \& PlunK, G.G. 2013 Collisionless microinstabilities in stellarators. I. Analytical theory of trapped-particle modes. Phys. Plasmas 20 (12), 122505.

Hirshman, S.P., Shaing, K.C., van Rij, W.I., Beasley, C.O. \& Crume, E.C. 1986 Plasma transport coefficients for nonsymmetric toroidal confinement systems. Phys. Fluids 29 (9), 2951-2959.

Huang, Z., Edlund, E., Porkolab, M., Bähner, J.-P., Böttger, L.-G., Sehren, C.v., von Stechow, A. \& GRULKe, O. 2021 The Wendelstein 7-X phase contrast imaging diagnostic. J. Instrum. 16 (01), P01014.

Jenko, F., Dorland, W., Kotschenreuther, M. \& Rogers, B.N. 2000 Electron temperature gradient driven turbulence. Phys. Plasmas 7 (5), 1904-1910. 
Kinsey, J.E., WALtZ, R.E. \& CANDY, J. 2005 Nonlinear gyrokinetic turbulence simulations of ExB shear quenching of transport. Phys. Plasmas 12 (6), 062302.

Klinger, T., Andreeva, T., Bozhenkov, S., Brandt, C., Burhenn, R., Buttenschön, B., Fuchert, G., Geiger, B., Grulke, O., LAQuA, H.P., et al. 2019 Overview of first Wendelstein 7-X high-performance operation. Nucl. Fusion 59 (11), 112004.

Lin, L., Porkolab, M., Edlund, E.M., Rost, J.C., Fiore, C.L., Greenwald, M., Lin, Y., MikKelsen, D.R., TsujiI, N. \& Wukitch, S.J., 2009 Studies of turbulence and transport in Alcator C-Mod H-mode plasmas with phase contrast imaging and comparisons with GYRO. Phys. Plasmas 16 (1), 012502.

Maurer, M., Bañón Navarro, A., Dannert, T., Restelli, M., Hindenlang, F., Görler, T., Told, D., Jarema, D., Merlo, G. \& Jenko, F. 2020 GENE-3D: A global gyrokinetic turbulence code for stellarators. J. Comput. Phys. 420, 109694.

MYNICK, H.E. \& HitCHON, W.N.G. 1983 Effect of the ambipolar potential on stellarator confinement. Nucl. Fusion 23 (8), 1053-1059.

Pablant, N.A., Langenberg, A., Alonso, A., Beidler, C.D., Bitter, M., Bozhenkov, S., Burhenn, R., Beurskens, M., Delgado-Aparicio, L., Dinklage, A., et al. 2018 Core radial electric field and transport in Wendelstein 7-X plasmas. Phys. Plasmas 25 (2), 022508.

Plunk, G.G., Connor, J.W. \& Helander, P. 2017 Collisionless microinstabilities in stellarators. IV. The ion-driven trapped-electron mode. J. Plasma Phys. 83 (4), 715830404.

Plunk, G.G., Helander, P., Xanthopoulos, P. \& Connor, J.W. 2014 Collisionless microinstabilities in stellarators. III. The ion-temperature-gradient mode. Phys. Plasmas 21 (3), 032112 .

Plunk, G.G., Xanthopoulos, P., Weir, G.M., Bozhenkov, S.A., Dinklage, A., Fuchert, G., Geiger, J., Hirsch, M., Hoefel, U., JAKUbOWski, M., et al. 2019 Stellarators resist turbulent transport on the electron larmor scale. Phys. Rev. Lett. 122 (3), 035002.

Proll, J.H.E., Helander, P., Connor, J.W. \& Plunk, G.G. 2012 Resilience of quasi-isodynamic stellarators against trapped-particle instabilities. Phys. Rev. Lett. 108 (24), 245002.

Proll, J.H.E., Mynick, H.E., Xanthopoulos, P., Lazerson, S.A. \& Faber, B.J. 2016 TEM turbulence optimisation in stellarators. Plasma Phys. Control. Fusion 58 (1), 014006.

Proll, J.H.E., Xanthopoulos, P. \& Helander, P. 2013 Collisionless microinstabilities in stellarators. II. Numerical simulations. Phys. Plasmas 20 (12), 122506.

Rost, J.C., Lin, L. \& Porkolab, M. 2010 Development of a synthetic phase contrast imaging diagnostic. Phys. Plasmas 17 (6), 062506.

Smith, H.M., Beidler, C.D., Turkin, Y., Bozhenkov, S., Ford, O. \& Fuchert, G. 2019 Would the Wendelstein 7-X 'record discharge' have been possible in other magnetic configurations? In 22nd International Stellarator and Heliotron Workshop 2019 (ISHW 2019), Madison, WI.

Stechow, A.V., Grulke, O., Wegner, T., Proll, J.H.E., Alcusón, J.A., Smith, H.M., Baldzuhn, J., Beidler, C.D., Beurskens, M.N.A., Bozhenkov, S.A., et al. 2021 Suppression of core turbulence by profile shaping in Wendelstein 7-X. Phys. Rev. Lett. https://arxiv. org/abs/2010.02160.

Tsujit, N., Porkolab, M., Bonoli, P.T., Edlund, E.M., Ennever, P.C., Lin, Y., Wright, J.C., WUKITCH, S.J., JAEGER, E.F., GREEN, D.L., et al. 2015 Validation of full-wave simulations for mode conversion of waves in the ion cyclotron range of frequencies with phase contrast imaging in Alcator C-Mod. Phys. Plasmas 22 (8), 082502.

Turkin, Y., Beidler, C.D., MaAßBerg, H., Murakami, S., Tribaldos, V. \& WaKasa, A. 2011 Neoclassical transport simulations for stellarators. Phys. Plasmas 18 (2), 022505.

Windisch, T., Smith, H.M., Alcusón, J.A., Lechte, C., Beurskens, M., Bozhenkov, S.A., Carralero, D., Damm, H., Estrada, T., Fuchert, G., et al. 2019 W-band doppler reflectometry at Wendelstein 7-X: Diagnostic setup and initial results. In Proc. 14th Intl. Reflectometry Wksh. - IRW14 (Lausanne, May 2019) (ed. G.D. Conway). Swiss Plasma Center EPFL.

Windisch, T., Wolf, S., Weir, G.M., Bozhenkov, S.A., Damm, H., Fuchert, G., Grulke, O., Hirsch, M., KaspareK, W., Klinger, T., et al. 2018 Phased array Doppler reflectometry at Wendelstein 7-X. Rev. Sci. Instrum. 89 (10), 10H115. 
Wolf, R.C., Alonso, A., Äkäslompolo, S., Baldzuhn, J., Beurskens, M., Beidler, C.D., Biedermann, C., Bosch, H.-S., Bozhenkov, S., Brakel, R., et al. 2019 Performance of Wendelstein 7-X stellarator plasmas during the first divertor operation phase. Phys. Plasmas 26 (8), 082504 .

Xanthopoulos, P., Bozhenkov, S.A., Beurskens, M.N., Smith, H.M., Plunk, G.G., Helander, P., Beidler, C.D., Alcusón, J.A., Alonso, A., Dinklage, A., et al. 2020 Turbulence mechanisms of enhanced performance stellarator plasmas. Phys. Rev. Lett. 125 (7), 075001.

Xanthopoulos, P., Mynick, H.E., Helander, P., Turkin, Y., Plunk, G.G., Jenko, F., Görler, T., Told, D., Bird, T. \& Proll, J.H.E. 2014 Controlling turbulence in present and future stellarators. Phys. Rev. Lett. 113 (15), 155001.

Yokoyama, M., Maaßberg, H., Beidler, C.D., Tribaldos, V., Ida, K., Estrada, T., Castejon, F., Fujisawa, A., Minami, T., Shimozuma, T., et al. 2007 Core electron-root confinement (CERC) in helical plasmas. Nucl. Fusion 47 (9), 1213-1219.

Zocco, A., Xanthopoulos, P., Doerk, H., Connor, J.W. \& Helander, P. 2018 Threshold for the destabilisation of the ion-temperature-gradient mode in magnetically confined toroidal plasmas. J. Plasma Phys. 84 (1), 715840101. 